\title{
Anatomical and Histological Studies of the Aqueous Outflow System in the Eye of Goat (Capra hircus)
}

\section{Ahmed Kassab ${ }^{1}$ and Ihab El-Zoghby ${ }^{2}$}

${ }^{1}$ Department of Anatomy and Embryology $\&^{2}$ Department of Histology and Cytology Faculty of Veterinary Medicine, Benha University (Moshtohor), Egypt.

Received July, accepted for publication August 2010

\section{Abstract}

The morphology of the goat aqueous outflow system and the iridocorneal anglewas studied in fifteen normal eyes from adult apparently healthy goats using light and scanning electron microscopy. The iridocorneal angle of the goat includes the pectinate ligament, the ciliary cleft, the trabecular meshwork (uveal and corneoscleral) and the angular aqueous plexus. The iridocorneal angle of the eye of goat was large; its length measured 1.3$1.7 \mathrm{~mm}$ and its width measured 1.0$1.2 \mathrm{~mm}$ anteriorly and 0.20-0.27 $\mathrm{mm}$ posteriorly. The pectinate ligamentwas a strong, band-like structure extending from the iridal base to the limbic zone. Its length varied from $500-700 \mu \mathrm{m}$ and its thickness was $120-150 \mu \mathrm{m}$. There were spaces and pores between its strands. The ciliary cleft contained a large amount of trabecular tissue that could be divided into two distinct parts: the uveal meshwork and the corneoscleral meshwork. The angular aqueous plexus consisted of

J. Vet. Anat. three to four endothelial-lined vessels or veins, located between the corneoscleral meshwork and the sclera. The results of this study revealed that the incidence of glaucoma in goat might be very rare.

\section{Key words}

Goat; Aqueous outflow system; Iridocorneal angle; Eye; Anatomy and Histology

\section{Introduction}

The aqueous fluid is produced by the cells of the ciliary body and reabsorbed through the filtration or drainage angle (iridocorneal angle) of the eye. Disorders in the region of the ciliary body and the iridocorneal angle are associated with changes in intraocular pressure (IOP). Thus the anatomical features of the aqueous humor outflow system (AOS) are clinically important in animals as a way to understand the etiology and pathogenesis of the 
many forms of glaucoma (McMenamin and Steptoe, 1991).

Many animals have been used as models in functional studies. The anatomy of the AOS has been investigated extensively in humans (Gierek, et al., 1974;Segawa, 1972 and Hogan, et al.,1971), rabbits (Ruskell, 1961and Bergmanson, 1985), horses (De et al., 1990 and Samuelson, et al. 1989), pigs (De, et al., 1987 and McMenamin and Steptoe, 1991) and dogs (Martin, 1975; Bedford and Grierson, 1986, and Samuel-son and Gelatt, 1984 a\&b). However, only a few papers have reported the structure of the AOS in ruminants (Anderson, et al., 1980 and Kassab, et al 2001). There are no available reports regarding the details of the AOS of the goat.

The pectinate ligament extends from the root of the iris to the inner aspect of the peripheral cornea. The pectinate ligament is rudimenttary in human and primates but it is highly developed in other animals, especially herbivores (Costa-Vila, et al. 1987 and Simones, et al. 1996). It had been studied in horses, donkeys, oxen, sheep, dogs, cats, pigs and rabbits using both light and scanning electron microscopes (De Geest, et al., 1987 and 1990 and Simones, et al., 1996)but there are no studies on the goat.

Therefore, the aims of the present study were: first, to provide a detailed description of the morphology of the goat aqueous outflow system and the iridocorneal angle in order to determine whether it may be a more appropriate model than some of the species currently in use. Second, to compare the structure of the goat's iridocorneal angle and pectinate ligament with that of other domestic animals and then to use these results as a basis for studying the morphological changes associated with the pathogenesis of primary open angle glaucoma.

\section{Materials and Methods}

Fifteen normal eyes from adult apparently healthy goats in various ages and sexes were used in this work. The eyes were obtained from Benha and Toukh slaughterhouses. The eyeballs were enucleated immediately after slaughter and immersed in a $10 \%$ formaldehyde solution buffered with phosphate buffer $(\mathrm{pH}$ 7.4) for fixation. A small cut was made in the cornea to allow this solution to penetrate inside the eyeball to insure good fixation of the inner structures.

Eight eyes were used for light microscopic study. The eyeballs were divided midway between the equator and the limbus and the lens was removed. The anterior half of the eye was subdivided into different pie-shaped segments of 2 or $3 \mathrm{~mm}$ in thickness. These segments were put in $10 \%$ buffered neutral formaldehyde solution, then dehydrated and embedded in paraffin. Frontal sections of 5-6 $\mu \mathrm{m}$ in thickness were obtained and stained with haematoxylin and Eosin (Harris, 
1898) and Crossmon's stains (Crossmon, 1937).

For scanning electron microscopy, seven eyes were used and dissectted into smaller blocks, dehydrated, critical point dried, further dissected if necessary, mounted, sputter coated with gold and viewed on a JEOL JSM 5500 LV SEM. The scanning electron microscopy was done at the Faculty of Agriculture, Alazhar University.

The length and width of the iridocorneal angle and the pectinate ligament were measured using SemAfore software.

The nomenclature used in this study was that of Nomina Anatomica Veterinaria and Nomina Veterinaria Histologica (1994).

\section{Results}

\section{The morphology of the irido- corneal angle}

The structures of the aqueous outflow system in the goat are present at the corneoscleral limbus of the eye at the iridocorneal angle (Angulus iridocornealis). The term iridocorneal angle describes the structures associated with the base of the iris, the anterior ciliary body, the inner of the cornea and sclera (Figs. 1 and 2). The iridocorneal angle of the eye of the goat was large and triangular. Its length measured 1.3$1.7 \mathrm{~mm}$ and its width measured 1.0$1.2 \mathrm{~mm}$ anteriorly and $0.20-0.27$ $\mathrm{mm}$ posteriorly (Fig. 2). The goat iridocorneal angle included the following structures: the pectinate ligament, the ciliary cleft, the trabecular meshwork (uveal and corneoscleral) and the angular aqueous plexus (Figs. 1, 2 and 3).

\section{The pectinate ligament (Liga- mentumpectinatum)}

The pectinate ligament (Figs. 1-5) was located on the anterior part of the iridocorneal angle. It was a strong, band-like structure extending from the iridal base to the limbic zone. Its length varied from $500-$ $700 \mu \mathrm{m}$ and its thickness was 120$150 \mu \mathrm{m}$. It contained consider-able amounts of melanin pigment, especially on the anterior border (Fig. 5). It was composed of many perforated collagenous sheets or lamellae (Fig. 10). The core of the pectinate ligament had a more compact arrangement because it contains more collagen fibers and less cellular material. There were spaces and pores between the strands of the pectinate ligament. The width of these spaces ranged between 10$23 \mu \mathrm{m}$. Under the SEM, the width of the pectinate ligament decreased along its course from the iris to the cornea (Fig. 6). The posterior strands of the pectinate ligament came together with the trabecular meshwork of the ciliary cleft.

\section{The ciliary cleft (sinus ciliaris)}

The ciliary cleft is the space bounded anteriorly by the pectinate ligament, externally by the limbic zone and internally by the base of the iris and the ciliary body (Figs. 2 
and 3). It was large, measuring 1.3$1.7 \mathrm{~mm}$ and $1.0-1.2 \mathrm{~mm}$ in height. It contained the trabecular meshwork.

The trabecular meshwork (reticulum trabeculae)

The goat eye contained a large amount of trabecular tissue that could be divided into two distinct parts: (a) the uveal part and (b) the corneoscleral part (Figs. 4-6).

\section{(a) The uveal meshwork (UM)}

The uveal meshwork is the inner part of the trabecular meshwork (Figs. 2, 3, 5, 6 and 7). It was composed of numerous strands or round trabeculae of various thicknesses $(15-35 \mu \mathrm{m})$. These trabeculae were thicker anteriorly and became thin and loose posteriorly. Melanin pigment was abundant along the uveal meshwork and near the stroma of the iris (Figs. 5). There were intertrabecular spaces between the trabeculae (Figs. 8). The width of the spaces was 25-45 $\mu \mathrm{m}$ and decreased gradually until they became very narrow posteriorly. There were also many round trabeculae in the anterior part of the ciliary cleft. Their intertrabecular spaces might be elongated and rounded.

\section{(b) The corneoscleral meshwork (CSM)}

The corneoscleral meshwork is the outer part of the trabecular meshwork (Figs. 2, 3, 5, 6 and 7). It was located between the sclera externally and the uveal trabeculae internally. It was extended from just

J. Vet. Anat. behind the pectinate ligament and continued posteriorly. The intertrabecular spaces of the corneoscleral meshwork were very small $(2-5 \mu \mathrm{m})$. The trabeculae of the corneoscleral meshwork were very thin, compact and were closely arranged. Dense collagen fibers were scattered.

\section{The angular aqueous plexus (AAP)}

The AAP consisted of three to four endothelial-lined vessels or veins (Fig. 5, 6, 7 and 9). The anterior one was the largest one of these veins (Fig. 5). These veins were found between the outer border of the corneoscleral meshwork and the sclera. The width of the trabecular vein was $5.0-8.0 \mu \mathrm{m}$. There was also some melanin pigment on the margin of the veins. The vessels of the AAP drain by interconnected collector channels (Fig. 9). The intrascleral venous plexus could be observed.

\section{Discussion}

The present study explains the features of the goat outflow system, which helps consequently in understanding the pathogenesis of some diseases like glaucoma in goats.

The general structure of the goat outflow system was similar to that of other domestic animals (Bedford and Grierson, 1986;De et al. 1987 and 1990; Kassab, et al. 2001; Samuelson and Lewis, 1995. 
The ciliary cleft of the goat iridocorneal angle is large, similar to that recorded in the horse (De Geest, et al.1990) and in contrast to that observed in pigs (McMenamin and Steptoe, 1991) and buffaloes (Kassab, et al., 2001). This large cleft facilitates the drainage of the aqueous humor in the goat iridocorneal angle. The smaller iridocorneal angles in primates and carnivores have been proposed to be the result of a concomitant enlargement of the ciliary body musculature (Samuelson et al., 1989 and Tripathi, 1974)

The pectinate ligament of the goat appeared as compact and strong, which is in good agreement with the reports of this structure in the buffalo (Kassab, et al., 2001) and bison (Samuelson and Lewis, 1995). On the other hand, the pectinate ligament was found to be a fenestrated sheet in horses (De Geest, et al., 1987 and 1990), cows (Samuelson and Lewis, 1995), dogs (Martin, 1975), pigs (De Geest, et al. 1987) and rabbits (Ruskell, 1961 and Simones, et al. 1996). So, the structure and shape of the goat pectinate ligament support the iridal base and prevent the collapse of the ciliary cleft.

The corneoscleral trabecular meshwork extended along the whole length of the outer wall of the ciliary cleft, like that in buffaloes (Kassab, et al. 2001), pigs (Mc Menamin and Steptoe, 1991) and dogs (Samuelson and Gelatt, 1984a). De Geest et al. (1990) has shown that in horses it occupies only the posterior angle of the ciliary cleft.

The present results also revealed that the UM and CSM came in contact with the posterior surface of the pectinate ligament, which is in agreement with that reported in buffaloes by Kassab, et al. (2001) and in bison and pigs by Samuelson and Lewis (1995). On the other hand, Samuelson and Lewis (1995) observed that the CSM was inserted at about $5 \mathrm{~mm}$ posterior to the pectinate ligament in the sheep and horses. So, the arrangement of these CSM may help in keeping the iridocorneal angle from collapse and consequently decrease the incidence of open angle glaucoma in the goat.

Tripathi (1974) mentioned that the aqueous humor exits through the iridocorneal angle via the AAP and intrascleral plexus (conventional route) and the uveoscleral route (unconventional route). Since trabecular tissues are abundant in the iridocorneal angle of the goat, there may be little resistance to the aqueous outflow along the conventional route at the corneoscleral meshwork. Similar results have been noted in cattle and horses (Huggert and Esklund, 1958 and Samuelson, et al. 1989). Therefore the uveoscleral pathway (unconventional route) may play a small role in the removal of the aqueous humor in goats. This latter opinion is in concurrence with that of Smith et al. (1986) and Samuelson et al. (1989) in horses. 
The results concerning the goat angular drainage vessels simulate the reports that were recorded in ungulates (Samuelson and Lewis, 1995), buffaloes (Kassab, et al. 2001), pigs (De Geest et al., 1987), horses (De Geest et al., 1990), cats (Richardson, 1982), dogs, ox, and rabbits (Samuelson and Gelatt, 1984b, Tripathi, 1974) and with the canal of Schlemm in primates (Tripathi, 1971, 1973 and 1974).

Since the angular aqueous plexus in the goat is composed of three to four obvious veins, it is likely that these trabecular veins are sufficient for the regular removal of the aqueous humor from the anterior chamber of the eye (conventional route).

In conclusion, this study describes the anatomical features of the aqueous outflow pathways in the goat. The morphology of the iridocorneal angle in the goat means that the incidence of glaucoma in the goat is probably very rare, as it is in buffaloes (Kassab, et al., 2001)and horses (De Geest et al., 1990).

\section{References}

Anderson, P.J., Wang, J., Epestein, D.L., (1980): Metabolism of calf trabecular (reticular) meshwork. Invest. Ophthalm. Vis. Sci. 19, 13-20.
Bedford, P.G., Grierson, I., (1986): Aqueous drainage in the dog. Res. Vet. Sci. 41, 172-186.

Bergmanson, J.P., (1985): The anatomy of the rabbit aqueous outflow pathway. ActaOphthalmologica.63, 493- 501.

Costa-Vila, J., Barastegui, C., Ruano-Gil, D., (1987): Morphological significance of the pectineal ligament of the eye. Acta Anat. 130, 247-250.

Crossmon, G., (1937): A modifycation of mallory connective tissue stain with discussion of the principle involved. Anat. Rec. 69, 33-38.

De Geest, J.P., Simones, P., Lauwers, H., De Schaepdrijver, L., (1987): The morphology of the iridocorneal angle of the pig eye. A light microscopic study .Zbt. Vet. Med. Reihe. C Anat. Hist. Embryol. 16, 245-249.

De Geest, J.P., Simones, P., Lauwers, H., De Schaepdrijver, L., (1990): The morphology of the iridocorneal angle of the equine eye. A light and scanning electron microscopic study.Equine Vet. J. 10, 30-35.

Gierek, A., Sosniez, M., Bialas, B. and Szymanski, A., (1974): Morphological picture of the iridocorneal angle of the human eyeball viewed under a scanning electron microscope. Ophthalmologia. 169, 371-376.

Harris, H. F., (1898):A new method of ripening hematoxylin. Cited by Carson (1997).

Hogan, M.J., Ararado, J.A., Weddle, J.E., (1971): Histology of 
the human eye. W.B. Saunders CO., Philadelphia, U.S.A. Huggert, A. and Esklund, A., (1958): The location of the chief obstruction to the outflow of aqueous humor from the eye of cattle. Acta.Ophth. 36, 50-56.

Nomina Anatomica Veterinaria (1994):International Committee on Veterinary Gross Anatomical Nomenclature of the World Association of Veterinary Anatomists, 4th Ed. Zurich and Ithaca, pp. 92-95. Together with Nomina Histologica Veterinaria, Organum visus.In Nomina Anatomica, pp. 29-31.

Kassab A, Aoyama M, Sugita S., (2001): The morphology of the iridocorneal angle in the eye of buffaloes (Bos bubalis): A light and scanning electron microscopic study. Okajimas Folia Anatomica Japonica. 78, 145152.

Martin, C.L., (1975): The normal canine iridocorneal angle as viewed with the scanning electron microscope. J. Am. Anim. Hosp. Ass. 11, 180-184.

McMenamin, P.G., Steptoe, R.J., (1991): Normal Anatomy of the Aqueous Outflow system in the Domestic pig Eye. J. Anat. 178, 65-77.

Richardson, T.M., (1982): Distribution of glycosaminoglycans in the aqueous outflow system of the cat. Invest Ophthalm. Vis. Sci. 22, 319-329.

Ruskell, G.L., (1961): Aqueous drainage paths in the rabbit. Arch. Ophth. 66, 861-870.
Samuelson, D.A., Gelatt, K.N. (1984a): Aqueous outflow in the beagle. I. Postnatal morphologic development of the iridocorneal angle: pectinate ligament and uveal trabecular meshwork. Current Eye Res. 3, 783-794.

Samuelson, D.A., Gelatt, K.N. (1984b): Aqueous outflow in the beagle. II. Postnatal morphologic development of the iridocorneal angle: corneoscleral trabecular meshwork and angular aqueous plexus. Current Eye Res. 3,795-807.

Samuelson, D. A., Lewis, P.A., (1995): Comparative morphology of the iridocorneal angle among selected Artiodactyls (ungulates). Vet. and Comp. Ophthal. 5, 89-103.

Samuelson, D.A., Smith, P.G., Brooks, D.E., (1989): Morphological features of the aqueous humor drainage pathways in horses. Am. J. Vet. Res. 50, 720-727.

Segawa, K., (1972): Scanning electron microscopic studies on the iridocorneal angle tissue in normal human eyes. Nippon. Ganka.Gakki.Zasshi. 76, 659663.

Simones, P., De Geest, J.P., Lauwers, H., (1996): Comparative morphology of the pectinate ligaments of domestic mammals, as observed under the dissecting microscope and the scanning electron micro-scope. J. Vet. Med. Sci. 58, 977-982.

Smith, P. J., Samuelson, D.A. Brooks, D. E. and Whitley, R.D., (1986):Unconventional aqueous 
humor outflow of microspheres perfused into the equine eye. Am. J. Vet. Res. 47, 2445-2453. Tripathi, R.C., (1971): Mechanism of the aqueous outflow across the trabecular wall of the Schlemm's canal. Exp. Eye. Res. 11, 116-121.

Tripathi, R.C., (1973): Comparative aspects of the aqueous outflow mechanism in primates, lower mammals and birds. Biorheology. 10, 498-499.

Tripathi, R.C., (1974): Comparative aspects of aqueous outflow. In The Eye. H. Davson and L. T. Graham (Eds). Academic Press, New York, pp. 163-356.
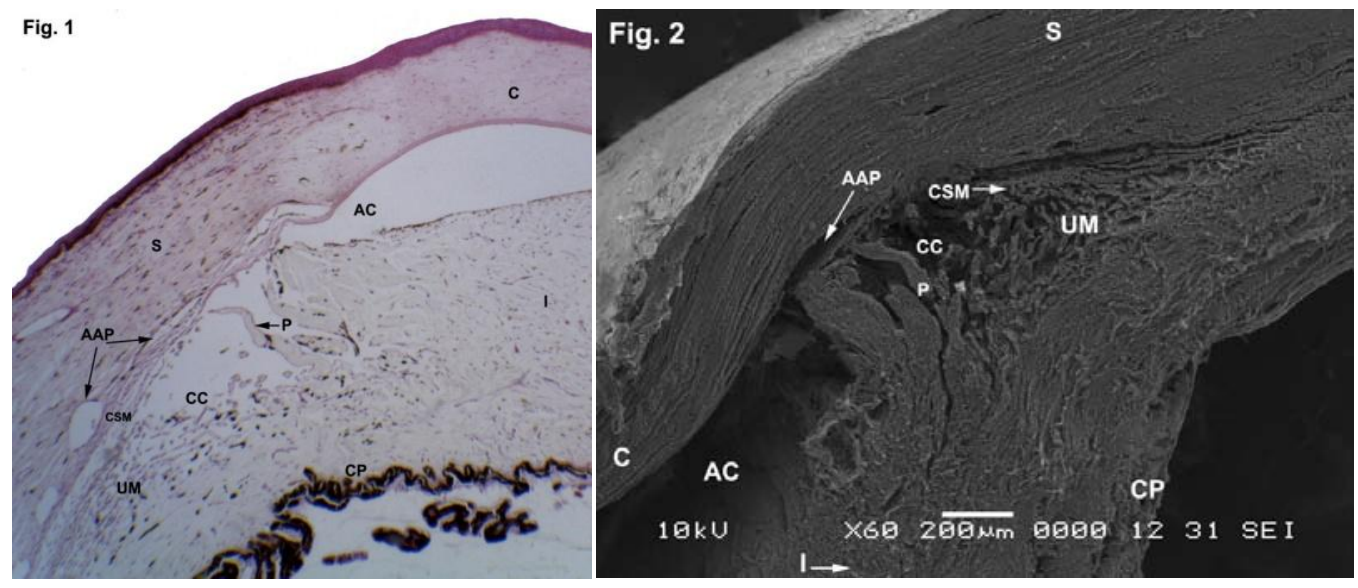

Fig (1): Photomicrograph of frontal section of the goat iridocorneal angle as seen with light microscope stained with H\&E stain. AC: anterior chamber, CC: ciliary cleft, CP: ciliary processes, CSM: corneoscleral meshwork, P: pectinate ligament, UM: uveal trabeculae, AAP: angular aqueous plexus, C: cornea, I: iris and S: sclera. X10.

Fig (2): Scanning electron micrograph showing the entire iridocorneal angle in the goat. AC: anterior chamber, CC: ciliary cleft, CP: ciliary processes, CSM: corneoscleral meshwork, P: pectinate ligament, UM: uveal trabeculae, AAP: angular aqueous plexus, C: cornea, I: iris and S: sclera. 

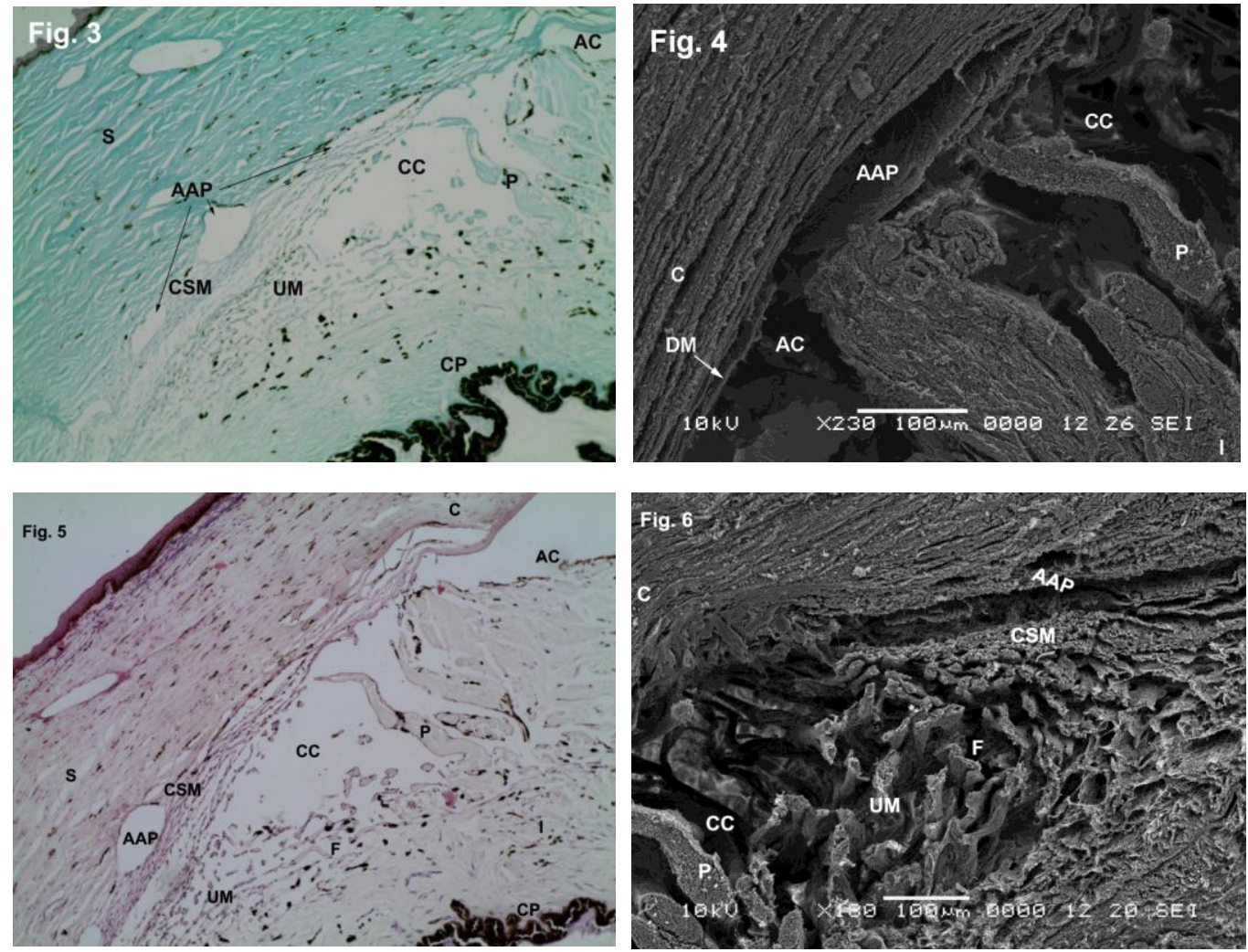

Fig (3): Photomicrograph of frontal section of the goat trabecular meshwork stained with Crossmon's stain. Abbreviations are the same in Fig. 1. X40

Fig (4): Medium power scanning electron micrograph showing the morphology of the pectinate ligament $(P)$, which merges with the corneal stroma $(C)$ and Descemet's membrane (DM) on one side of the ciliary cleft (CC) and the iris (I) on the other side.

Fig (5): High magnification of the goat iridocorneal angle stained with H\&E stain showing the intertrabecular spaces (spaces of Fontana): $F$ and the difference between the uveal and corneoscleral meshworks. Abbreviations are the same in Fig. 1. X40

Fig (6): Scanning electron micrograph showing the goat trabecular meshwork. Note the arrangement of the collagen fibers in the uveal and corneoscleral trabeculae. Abbreviations are the same in Fig. 1. 

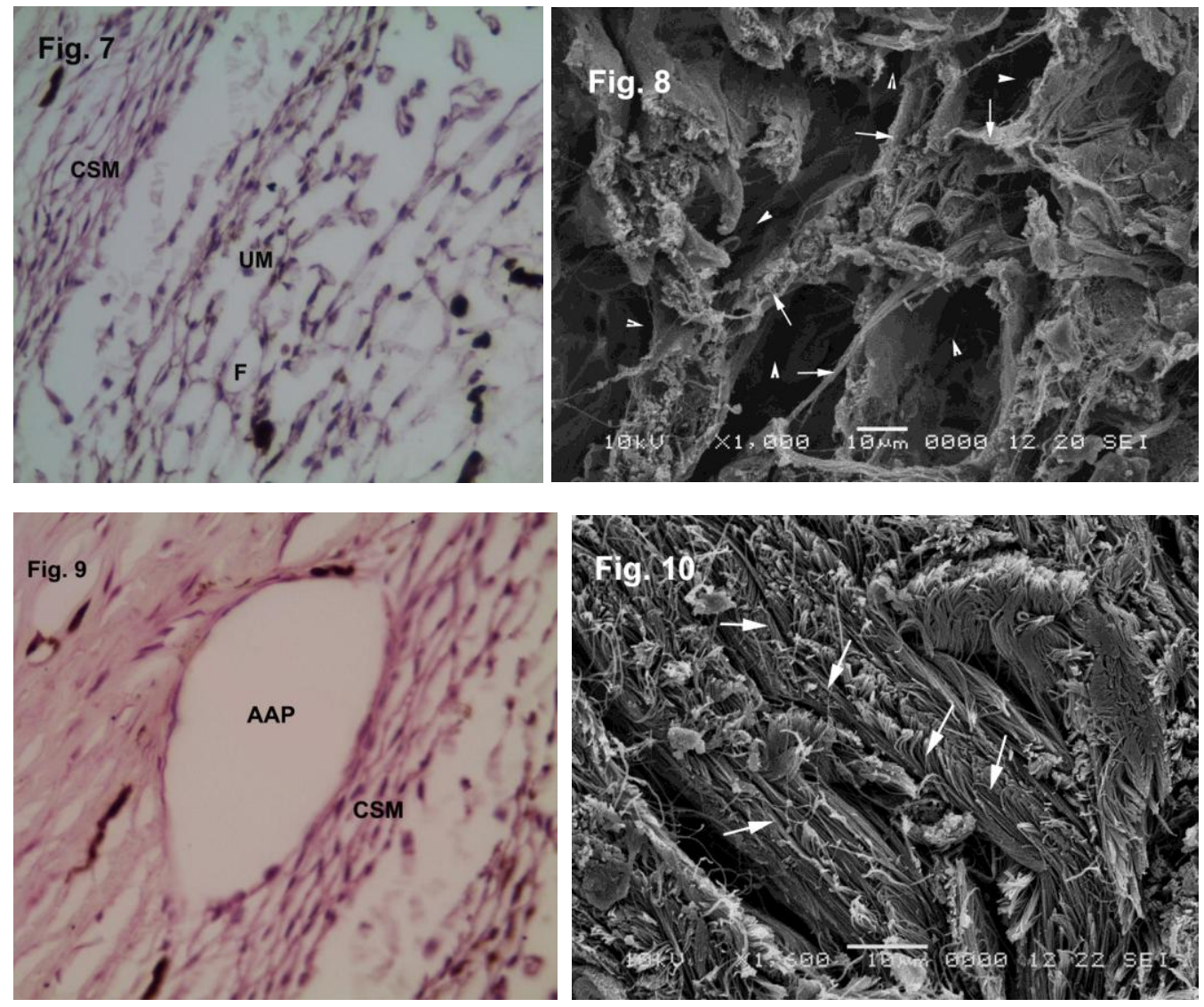

Fig (7): Photomicrograph of well-organized trabeculae in the uveal and corneoscleral meshwork. CSM: corneoscleral meshwork, UM: uveal trabeculae, F: spaces of Fontana. H\&E X40.

Fig (8): Scanning electron micrograph of goat uveal meshwork showing the arrangement of the collagen fibers in the trabeculae (arrows) and the intertrabecular spaces (arrow heads).

Fig (9): High magnification of the outer meshwork adjacent to the angular aqueous vessels. AAP: angular aqueous plexus and CSM: corneoscleral meshwork. H\&E. X400

Fig (10): High power scanning electron micrograph of the goat pectinate ligament showing perforated collagenous sheets or lamellae (arrows). 